\title{
Undernutrition, intestinal parasitic infection and associated risk factors among selected primary school children in Bahir Dar, Ethiopia
}

Tamirat Hailegebriel

\begin{abstract}
Background: Monitoring of undernutrition and parasitic infection are essential to design appropriate intervention strategies. The aim of this study was to assess the prevalence of undernutrition, intestinal parasitic infection and their associated risk factors among school children in Bahir Dar, Ethiopia.

Methods: A school-based cross-sectional survey was conducted from February to June 2014 among 382 students selected from primary schools in Bahir Dar. The study subjects were selected by a systematic random sampling method. Sociodemographic data from students and their family/guardians were obtained using structured questionnaire. Height and weight of the students were measured using a standard calibrated balance. Fresh fecal samples were collected and processed using formalin-ether concentration technique. The data were analyzed using SPSS version 20.0 statistical software.

Results: The overall prevalence of undernutrition was $41.6 \%$ (18.3\% stunted, 26.7\% thinness and 25.9\% underweight). Meal frequency $\leq 3$ times a day ( $\mathrm{AOR}=4.11 ; 95 \% \mathrm{Cl}: 2.23-7.59)$ and family monthly income $<500$ birr ( $A O R=5.87 ; 95 \% \mathrm{Cl}: 2.61-13.23$ ) were important predictors of undernutrition. The risk of stunting was increased among students with meal frequency $\leq 3$ times a day ( $\mathrm{AOR}=5.56 ; 95 \% \mathrm{Cl}: 2.97-10.41)$ and age ranges from 9-10 years (AOR=3.02; 95\% Cl: 1.41-6.47). The odds of thinness was significantly increased among students with parasitic infection ( $A O R=1.92 ; 95 \% \mathrm{Cl}: 1.15-3.19$ ) and family monthly income $<1500$ birr (AOR=2.69; 95\% Cl: 1.16-6.26). The likelihood of being underweight was increased among students infected with intestinal parasites (AOR=2.43; 95\% Cl: 1.40-4.22). The overall prevalence of intestinal parasitosis was 52.4\%. The risk of parasitic infection was significantly increased among students with unclean fingernails (AOR=4.96; 95\% Cl: 2.79-8.82) and irregular hand washing habit (AOR=8.05; 95\% Cl: 4.66-13.89).

Conclusions: This study revealed that undernutrition and intestinal parasitic infection were public health problems among school children in the study areas. These results highlight the importance for integrated efforts to address undernutrition and parasitic infection.
\end{abstract}

Keywords: Children, parasitic infection, undernutrition, stunting, underweight, thinness

(C) The Author(s). 2018 Open Access This article is distributed under the terms of the Creative Commons Attribution 4.0 International License (http://creativecommons.org/licenses/by/4.0/), which permits unrestricted use, distribution, and reproduction in any medium, provided you give appropriate credit to the original author(s) and the source, provide a link to the Creative Commons license, and indicate if changes were made. The Creative Commons Public Domain Dedication waiver (http://creativecommons.org/publicdomain/zero/1.0/) applies to the data made available in this article, unless otherwise stated. 


\section{Background}

Undernutrition is one of the major causes of child mortality and morbidity in developing countries. Globally, 104 million and 171 million children are stunted and underweight in 2010, respectively [1]. The problem is particularly severe in Africa where $20.2-48.1 \%$ of preschool children are stunted and 14-36.5\% are underweight [2]. Undernutrition is manifested by protein-energy malnutrition, iodine deficiency disorder, iron deficiency anemia, and vitamin deficiency related problems. The combined effects of these problems have an immense impact on children health, growth, development, and academic performance. Undernutrition is further aggravated by the presence of intestinal parasitic infections.

Intestinal parasitic infections are major health problems in preschool and school children in many developing countries. According to World Health Organization (WHO), more than 1.5 billion peoples are infected with soil-transmitted helminthes and 870 million children are living in areas where parasitic worm infection is endemic[3]. The majority of this burden is concentrated in developing countries mainly in Africa and Asia. For instance, more than $50 \%$ of school children in Sub-Saharan Africa are infected with soil-transmitted helminthes [4]. This high burden of helminthic infections further leads to undernutrition through reduced food intake, malabsorption, endogenous nutrient loss and anemia related nutritional problem via excessive blood cell destruction [5]. The presences of hook worm, Schistosoma mansoni, Trichuris trichura and Ascaris lumbricoides infection in particular are associated with undernutrition [6-8]. Undernutrition and helminthic infections are overlapping in many developing countries and may have a negative effect on student growth and development as well as their academic performance [9].

Ethiopia is one of the Sub-Saharan-African countries with high prevalence of intestinal parasitic infections and undernutrition. The prevalence of undernutrition and intestinal parasitic infections are varied from region to region. The overall prevalence of undernutrition in first cycle primary school children in Ethiopia ranges from 11-50\% [10-13]. Likewise, the prevalence of intestinal parasitic infections in school children are ranging from $27.2-72 \%[12,14,15]$ as reported from several parts of the country. Co-occurrence of undernutrition and parasitic infections are not uncommon in the country. For example, about $22.4 \%$ of school children were undernourished as well as infected by parasitic organism in Adama, Ethiopia [10].

Epidemiological survey on the prevalence of undernutrition and intestinal parasitic infection is essential to develop appropriate intervention strategies. However, information on the nutritional status, intestinal parasitic infection and associated risk factors among school children in the study area is lacking. Therefore, this study aimed to assess the nutritional status, prevalence of intestinal parasitic infection, and their associated risk factors among first cycle primary school children in Bahir Dar, Ethiopia.

\section{Methods \\ Study design and study area}

A school-based cross-sectional study was conducted from February to June 2014 among students in selected first cycle primary schools at Bahir Dar, Ethiopia. Three government schools were systematically selected from the northern, central and southern part of Bahir Dar city. The city is located at $11^{\circ} 6^{\prime} \mathrm{N}, 37^{\circ} 38^{\prime} \mathrm{E}, 1784$ meter above sea level and $578 \mathrm{~km}$ distant from Addis Ababa, the capital city of Ethiopia. The northern part of the city is surrounded by Lake Tana, which is the largest lake in Ethiopia and the source of the Blue Nile River. The Blue Nile River cross the city and then surrounds the eastern part. Bahir Dar is one of the fast-growing cities in the country and the administrative center of Amhara regional state. According to 2007 Ethiopian population census, the total population of the city is about 180,094 with the majority of them are children[16].

\section{Study population and sample size determination}

The study participants were students attending at the selected primary schools (Teyma, Dilchibo and Dona Berber) from grade 1 to grade 4 . A total of 56 classes were available in the selected schools and each class contains an average of 64 students during the study period. The schools were selected based on their representativeness in the city (northern part, central part and southern part of the city). The total numbers of students attending in the three primary schools were 3584 at study period. Students were selected by stratified sampling based on their educational level (grade 1 to grade 4) and then quota was allocated to each grade based on the total number of students. The target students were selected by systematic random sampling from each class based on their class roster.

The study populations were determined by a statistical formula used for sample size determination: $n=Z^{2} x P$ $(1-\mathrm{P}) / \mathrm{d}^{2}$ where: $\mathrm{Z}$ at $95 \%$ confidence interval, $\mathrm{P}$ at $50 \%$ (the prevalence of undernutrition was not known), $d$ at $5 \%$ marginal error and 1-p (non-observed value). Based on this information, the study populations defined were 384 children. To avoid errors due to non-response rate, the numbers of students selected for the study were increased by $10 \%$. Therefore, the total numbers of students selected for the present study were 422 .

\section{Collections of sociodemographic information}

Socio-demographic variables of students and family/guardians of each student involved in the study were collected through structured questionnaires. The questionnaires were 
prepared in English and then translated to Amharic (local language in the study area). The age of the students was taken from registration book of the school and then confirmed from their parents/guardians. The habits of using protective shoe and washing their hand before meal were asked by the data collectors. In the meantime, finger cleanness (the presence or absence of dirty material in their finger nail) and their overall personal hygiene of each student were checked by the data collector. The data collection was performed by trained individuals having experience in similar work under continuous supervision by the investigator.

\section{Anthropometric measurement and nutritional status assessment}

Anthropometric measurements were taken by considering sex, age, height and weight of the students. A portable battery powered digital balance was used to measure weight and height of each student to the nearest $0.1 \mathrm{~kg}$ and $0.1 \mathrm{~cm}$, respectively. The height and weight measurements were taken in a minimum cloth and without shoe. Two consecutive measurements were taken from each student and an average of the two measurements was used to calculate the anthropometric indices. The digital balance was calibrated after each measurement.

The anthropometric indices were determined based on 2007 WHO growth reference data for children and adolescence [17]. Z score value for each student was calculated using Anthro-Plus software based on student sex, age, height, and weight [18]. The nutritional status of students was determined based on the information from $\mathrm{Z}$ score. The nutritional indicators were labeled as stunting, underweight and thinness based on height-for-age (HAZ), weight-for-age (WAZ) and body mass index-for-age (BAZ) [19], respectively. HAZ, WAZ and BAZ score less than - 2 standard deviations (SD) from WHO reference populations were considered as stunted, underweight and thin, respectively. If the $\mathrm{Z}$ score value was less than $-3 \mathrm{SD}$, the students categorized as severely undernourished for each indicator. WAZ measurements for children above 10 years of age were not performed in the study due to lack of WHO reference data and Anthro-Plus software was not applicable for these children. Children above 10 years of age may show secondary sexual characteristic that leads to increase their weight as their age increase. This change makes WAZ based assessment of nutritional status unsuitable for children above 10 years of age. Therefore, the numbers of students involved in WAZ assessment were less than students participated in HAZ and BAZ assessment.

\section{Parasitological examinations}

The stool specimens were collected and processed based on WHO guidelines. Each student was given a clean and labeled plastic vial with an applicator stick to bring about
2 grams of fresh stool sample. The collected stool samples were properly mixed with $10 \mathrm{~mL}$ of $10 \%$ formalin for preservation. The preserved stool samples were processed using formalin-ether concentration techniques as described in WHO guidelines [20] by trained laboratory technologist. All the different developmental stages (eggs, cyst, oocyst, larvae, adult and segments of adult worm) of the parasitic organism were recorded.

\section{Quality control}

Among the total positive samples for parasitic infection, $10 \%$ of them were randomly selected, processed and examined by senior laboratory technologist who did not have information about the previous result. The result of this examination was used for quality control for parasitic examination in the study.

\section{Statistical analysis}

The collected data were analyzed using SPSS version 20 software. Z scores for the different anthropometric indices (HAZ, WAZ and BAZ) were calculated using WHO Anthro-plus version 1.0.4 software [18]. The association of potential risk factors with intestinal parasitic infection and undernutrition (stunting, underweight and thinness) were analyzed using binary logistic regression model and the degree of associations were expressed in odds ratio. The defined value of $\mathrm{p}<0.05$ were considered as statistically significant.

\section{Results}

\section{Socio demographic characteristics}

Out of the total 422 students invited in the study, 40 (9.5\%) students were excluded from the analysis due to dropout, incomplete information or insufficient stool sample size. A total of 382 primary school children age ranges from 7 to 13 years were eligible for the data analysis. The numbers of male and female students participated in the study were nearly equal (Table 1 ). The mean (SD) age of students participated in the study was $9.5( \pm 1.6)$ years.

\section{Prevalence of intestinal parasitic infection and associated risk factors}

Among the 382 students participated in the study, 200 (52.4\%) were positive for one or more intestinal parasites (Table 2). The rates of intestinal parasitic infections were 26.7 and $25.7 \%$ among male and female students, respectively. Double and triple infections were not uncommon; about 24 (6.3\%) of the study participants were infected by two or more intestinal parasites at a time. The most common intestinal parasitic infections detected in the study were E. histolytica/dispar (16.8\%), Hookworm infection (14.7\%) and A. lumbricoides (13.6\%) (Table 2).

According to multivariate logistic regression model, student with unclean fingernails (AOR=4.96; 95\% CI: 2.79- 
Table 1 Sociodemographic characteristic and nutritional status of school children and their family in selected primary school in Bahir Dar, Ethiopia, 2014

\begin{tabular}{|c|c|c|c|}
\hline Variables & Categories & Frequency & Percentage \\
\hline \multirow[t]{2}{*}{ Sex student } & Female & 189 & 49.5 \\
\hline & Male & 193 & 50.5 \\
\hline \multirow[t]{3}{*}{ Age of students } & $7-8$ & 118 & 30.9 \\
\hline & $9-10$ & 175 & 45.8 \\
\hline & $11-13$ & 89 & 23.3 \\
\hline \multirow[t]{3}{*}{ Family monthly income $\left(\mathrm{ETB}^{\mathrm{a}}\right)$} & $<500$ & 106 & 18.1 \\
\hline & $500-1500$ & 215 & 56.5 \\
\hline & $>1500$ & 61 & 25.4 \\
\hline \multirow[t]{3}{*}{ Mother education } & Illiterate & 125 & 32.5 \\
\hline & Primary school & 143 & 37.4 \\
\hline & Secondary school and above & 114 & 29.8 \\
\hline \multirow[t]{2}{*}{ Hand washing habit } & Sometimes & 170 & 44.5 \\
\hline & Always & 212 & 55.5 \\
\hline \multirow[t]{2}{*}{ Shoe wearing habit } & Sometimes & 201 & 52.6 \\
\hline & Always & 181 & 47.4 \\
\hline \multirow[t]{3}{*}{ Religion } & Orthodox & 265 & 69.4 \\
\hline & Muslim & 49 & 12.8 \\
\hline & Protestant & 68 & 17.8 \\
\hline \multirow[t]{2}{*}{ Finger cleanness } & Not clean & 117 & 30.6 \\
\hline & Clean & 265 & 69.4 \\
\hline \multirow[t]{2}{*}{ Meal frequency } & $\leq 3$ times a day & 288 & 75.4 \\
\hline & $>3$ times/day & 94 & 24.6 \\
\hline \multirow[t]{2}{*}{ Overall nutritional status } & $H A Z, B A Z \& W A Z<-2 S D$ & 159 & 41.6 \\
\hline & $H A Z, B A Z \& W A Z>-2 S D$ & 223 & 58.4 \\
\hline \multirow[t]{2}{*}{ Stunting } & $H A Z<-2 S D$ & 70 & 18.3 \\
\hline & $\mathrm{HAZ}>-2 \mathrm{SD}$ & 312 & 81.7 \\
\hline \multirow[t]{2}{*}{ Thinness } & $\mathrm{BAZ}<-2 \mathrm{SD}$ & 102 & 26.7 \\
\hline & $B A Z>-2 S D$ & 280 & 73.3 \\
\hline \multirow[t]{2}{*}{ Underweight } & $W A Z<-2 S D$ & 76 & 25.9 \\
\hline & WAZ>-2 SD & 217 & 74.1 \\
\hline \multirow[t]{2}{*}{ Parasitic infection } & Infected & 200 & 52.4 \\
\hline & Not infected & 182 & 47.6 \\
\hline
\end{tabular}

${ }^{\mathrm{a} E t h i o p i a n ~ B i r r ~}$

8.82), irregular hand washing habit (AOR=8.05; 95\% CI: 4.66-13.89), undernutrition ( $\mathrm{AOR}=1.69$; 95\% CI: $1.05-$ 2.74) and being borne from illiterate mother $(\mathrm{AOR}=2.00$, 95\% CI: 1.12-3.57) were independent predictors intestinal parasitic infection (Table 3). The presences of intestinal parasitic infections were independent on family monthly income, sex, age, and meal frequency of students.

\section{Prevalence of undernutrition and its associated risk} factors

Out of the 382 students participated in the study, 159 (41.6\%) was undernourished (18.3\% stunted, $26.7 \%$ thinness and 25.9\% underweight) (Table 1). Among those undernourished students, $45.9 \%$ were positive for two or more forms of undernutrition at a time. Binary logistic regression model showed that students borne from family with monthly income less than 1500 birr (AOR=5.51, 95\% CI: 2.45-12.40), meal frequency at most 3 times a day $(\mathrm{AOR}=4.11,95 \% \mathrm{CI}: 2.23-7.59)$ and presence of intestinal parasitic infection (AOR $=1.60 ; 95 \% \mathrm{CI}$ : 1.03-2.46) were strongly associated with undernutrition (Table 4). In the present study, undernutrition was strongly associated with $A$. lumbricodes but not hookworm infection $(p<0.05)$. 
Table 2 Prevalence of intestinal parasitic infection among children by sex in selected primary school in Bahir Dar, Ethiopia, 2014

\begin{tabular}{|c|c|c|c|c|c|}
\hline \multicolumn{2}{|c|}{ Parasite species } & \multicolumn{3}{|c|}{ Parasite infected students by sex } & \multirow[b]{2}{*}{ Chi-Square $P$-value } \\
\hline & & $\begin{array}{l}\text { Female } \\
\text { No. (\%) }\end{array}$ & $\begin{array}{l}\text { Male } \\
\text { No. (\%) }\end{array}$ & $\begin{array}{l}\text { Total } \\
\text { No. (\%) }\end{array}$ & \\
\hline \multirow[t]{4}{*}{ Protozoa } & Entamoeba histolytica/dispar & $30(7.9)$ & $34(8.9)$ & $64(16.8)$ & 0.65 \\
\hline & Giardia lamblia & $0(0)$ & $1(0.3)$ & $1(0.3)$ & 0.32 \\
\hline & Isospora belli & $0(0)$ & $1(0.3)$ & $1(0.3)$ & 0.32 \\
\hline & Enterobius vermicularis & $1(0.3)$ & $0(0)$ & $1(0.3)$ & 0.31 \\
\hline \multirow[t]{7}{*}{ Helminths } & Ascaris lumbricoides & $27(7.1)$ & $25(6.5)$ & $52(13.6)$ & 0.70 \\
\hline & Hookworm & $30(7.9)$ & $26(6.8)$ & $56(14.7)$ & 0.51 \\
\hline & Hymenoleps nana & $12(3.1)$ & $16(4.2)$ & $28(7.3)$ & 0.48 \\
\hline & Schistosoma mansoni & $6(1.6)$ & $3(0.8)$ & $9(2.4)$ & 0.29 \\
\hline & Trichuris trichiura & $4(1)$ & $3(0.8)$ & $7(1.8)$ & 0.68 \\
\hline & Strongyloides stericoralis & $1(0.3)$ & $4(1)$ & $5(1.3)$ & 0.19 \\
\hline & Taenia spp & $0(0)$ & $1(0.3)$ & $1(0.3)$ & 0.32 \\
\hline \multicolumn{2}{|c|}{ Single infection } & 85 (22.3) & $91(23.8)$ & $176(46.1)$ & 0.67 \\
\hline \multicolumn{2}{|c|}{ Multiple infection } & $13(3.4)$ & $11(2.9)$ & $24(6.3)$ & 0.64 \\
\hline \multicolumn{2}{|c|}{ Over all infection } & $98(25.7)$ & $102(26.7)$ & $200(52.4)$ & 0.85 \\
\hline
\end{tabular}

Table 3 Bivariate and multivariate logistic regression analysis of potential risk factors associated with parasitic infection among children in selected primary school in Bahir Dar, Ethiopia, 2014

\begin{tabular}{|c|c|c|c|c|c|c|}
\hline \multirow[t]{2}{*}{ Risk factors } & \multirow[t]{2}{*}{ Categories } & \multicolumn{2}{|c|}{ Parasitic infection } & \multirow[b]{2}{*}{ Total (\%) } & \multirow{2}{*}{$\begin{array}{l}\text { Crude OR (Cl } \\
95 \%)\end{array}$} & \multirow{2}{*}{$\begin{array}{l}\text { Adjusted OR (Cl } \\
95 \%)^{\mathrm{a}}\end{array}$} \\
\hline & & Yes (\%) & No (\%) & & & \\
\hline \multirow[t]{2}{*}{ Nutritional status } & Undernourished $(Z<-2 S D)$ & $71(18.6)$ & $88(23)$ & $159(41.6)$ & $1.70(1.13-2.56)^{*}$ & $1.69(1.05-2.74)^{*}$ \\
\hline & Normal (Z>-2SD) & $129(33.8)$ & $94(24.6)$ & $223(58.4)$ & 1 & 1 \\
\hline \multirow[t]{2}{*}{ Hand washing habit } & Sometimes & $149(39)$ & $63(16.5)$ & $212(55.5)$ & $5.52(3.55-8.58)^{* *}$ & $8.05(4.66-13.89)^{* *}$ \\
\hline & Always & $51(13.4)$ & $119(31.2)$ & $170(44.5)$ & 1 & 1 \\
\hline \multirow[t]{2}{*}{ Shoe wearing habit } & Sometimes & $115(30.1)$ & $86(22.5)$ & $201(52.6)$ & $1.51(1.01-2.26)^{*}$ & $1.02(0.61-1.70)$ \\
\hline & Always & $85(22.3)$ & $96(25.1)$ & $181(47.4)$ & 1 & 1 \\
\hline \multirow[t]{2}{*}{ Fingernail cleanness } & Not clean & $85(22.3)$ & $32(8.4)$ & 117 (30.6) & $3.46(2.16-5.56)^{* *}$ & $4.96(2.79-8.82)^{* *}$ \\
\hline & Clean & $115(30.1)$ & $150(39.3)$ & $265(69.4)$ & 1 & 1 \\
\hline \multirow[t]{3}{*}{ Mother education } & Illiterate & $76(19.9)$ & 49 (12.8) & $125(32.7)$ & $2.03(1.24-3.30)^{* *}$ & $2.00(1.12-3.57)^{*}$ \\
\hline & Primary school & $62(16.2)$ & $81(21.2$ & $143(37.4)$ & $1.30(0.78-2.18)$ & $1.56(0.84-2.90)$ \\
\hline & Secondary school \& above & $62(16.2)$ & $52(13.6)$ & $114(29.8)$ & 1 & 1 \\
\hline \multirow[t]{3}{*}{ Family monthly income (ETB ${ }^{@}$ ) } & $<500$ & $57(14.9)$ & $49(12.8)$ & $106(27.7)$ & $0.81(0.51-1.28)$ & \\
\hline & $500-1500$ & $104(27.2)$ & $111(29.1)$ & $215(56.3)$ & $1.52(0.79-2.91)$ & \\
\hline & $>1500$ & $39(10.2)$ & $22(5.8)$ & $61(16)$ & 1 & \\
\hline \multirow[t]{2}{*}{ Meal frequency } & $\leq$ 3times a day & $152(39.8)$ & $136(35.6)$ & $288(75.4)$ & $1.07(0.67-1.71)$ & \\
\hline & $>3$ times/day & $48(12.6)$ & $46(12)$ & $94(24.6)$ & 1 & \\
\hline \multirow[t]{2}{*}{ Sex of students } & Male & $102(26.7)$ & $91(23.8)$ & $193(50.5)$ & $0.96(0.64-1.44)$ & \\
\hline & Female & $98(25.7)$ & $91(23.8)$ & $189(49.5)$ & 1 & \\
\hline \multirow[t]{3}{*}{ Age (years) } & $7-8$ & $63(16.5)$ & $55(14.4)$ & $118(30.9)$ & $0.88(0.55-1.41)$ & \\
\hline & $9-10$ & $88(23)$ & $87(22.8)$ & $175(45.8)$ & $1.07(0.62-1.86)$ & \\
\hline & $11-13$ & $49(12.8)$ & $40(10.5)$ & 89 (23.3) & 1 & \\
\hline
\end{tabular}




\section{Factor associated with stunting}

Among the total students participated in the study, 70 (18.3\%) were stunted, of which $20 \%$ were severely stunted $(\mathrm{HAZ}<-3 \mathrm{SD})$. Students with meal frequency at most 3 times a day $(\mathrm{AOR}=5.56$; 95\% CI: $2.97-10.41)$, age ranges from 9-10 years old ( $\mathrm{AOR}=3.02$; 95\% CI: 1.416.47), and being borne from a mother with primary school education ( $\mathrm{AOR}=2.25$; 95\% CI: $1.06-4.78$ ) were strongly associated with increased odds of being stunted (Table 5). The presence of parasitic infection, family monthly income, hand washing habit and finger cleanness of students were not associated with stunting in this study.

\section{Factor associated with thinness}

Of the total participants, $102(26.7 \%)$ were showed the problem of thinness (Table 6). Among those students, $22(21.6 \%)$ were severely thinned $(\mathrm{BAZ}<-3 \mathrm{SD})$. Presence of intestinal parasitic infection (AOR $=1.92 ; 95 \% \mathrm{CI}$, $1.15-3.19)$ and belonging to a family with monthly income less than 1500 birr (AOR=2.69; 95\% CI, 1.16-6.26) were independently predicting thinness (Table 6).
Factor associated with underweight

A total of 293 students age ranges from 7-10 years were used to assess the prevalence of underweight. Seventy six students $(25.9 \%)$ were underweight of which 25 $(32.9 \%)$ were severely underweight (WAZ<-3SD). Binary logistic regression model showed that underweight were strongly associated with parasitic infection $(\mathrm{AOR}=2.43$; 95\% CI: 1.40-4.22), meal frequency at most three times a day (AOR=1.77; 95\% CI: $1.03-3.05$ ) and being a male in sex (AOR=1.76; 95\% CI: 1.02-3.04) (Table 7).

Co-occurrence of undernutrition and parasitic infections were common in the study population. Out of the total 159 undernourished students, 71 (44.7\%) were infected by one or more parasitic organism. This indicated that nearly half of the undernourished students were infected by intestinal parasites.

\section{Discussion}

Monitoring of intestinal parasitic infection and nutritional status of school children are essential to improve their health conditions and academic performance through appropriate intervention strategies. The present study revealed that more than half of school children

Table 4 Binary logistic regression analysis of potential risk factors associated with undernutrition among children at selected primary schools in Bahir Dar, Ethiopia, 2014

\begin{tabular}{|c|c|c|c|c|c|c|}
\hline \multirow[t]{2}{*}{ Risk factors } & \multirow[t]{2}{*}{ Categories } & \multicolumn{2}{|c|}{ Nutritional Status } & \multirow[b]{2}{*}{ Total (\%) } & \multirow{2}{*}{$\begin{array}{l}\text { Crude OR (Cl } \\
95 \%)\end{array}$} & \multirow{2}{*}{$\begin{array}{l}\text { Adjusted OR (Cl } \\
95 \%)^{\mathrm{a}}\end{array}$} \\
\hline & & Yes (\%) & No (\%) & & & \\
\hline \multirow[t]{3}{*}{ Family monthly income $\left(\mathrm{ETB}^{@}\right)$} & $<500$ & $53(13.9)$ & $53(15.9)$ & $106(27.7)$ & $3.36(1.65-8.81)^{* *}$ & $5.87(2.61-13.23)^{* *}$ \\
\hline & $500-1500$ & $92(24.1)$ & $123(32.3)$ & $215(56.3)$ & $2.51(1.30-4.83)^{*}$ & $5.51(2.45-12.40)^{* *}$ \\
\hline & $>1500$ & $14(3.7)$ & $47(12.3)$ & $61(16)$ & 1 & 1 \\
\hline \multirow[t]{2}{*}{ Meal frequency } & $\leq$ 3times a day & $106(27.7)$ & $182(47.6)$ & $288(75.4)$ & $2.22(1.38-3.56)^{* *}$ & $4.11(2.23-7.59)^{* *}$ \\
\hline & $>3$ times/day & $53(13.9)$ & $41(10.7)$ & $94(24.6)$ & 1 & 1 \\
\hline \multirow[t]{2}{*}{ Parasitic infection } & Infected & $71(18.6)$ & $129(33.8)$ & $200(52.4)$ & $1.70(1.13-2.56)^{*}$ & $1.60(1.03-2.46)^{*}$ \\
\hline & Not infected & $88(23)$ & $94(24.6)$ & $182(47.6)$ & 1 & 1 \\
\hline \multirow[t]{2}{*}{ Sex of students } & Male & $90(23.6)$ & $103(27)$ & $193(50.5)$ & $1.52(1.01-2.29)^{*}$ & $1.46(0.95-2.26)$ \\
\hline & Female & $69(18.1)$ & $120(31.4)$ & $189(49.5)$ & 1 & 1 \\
\hline \multirow[t]{3}{*}{ Age (years) } & $7-8$ & $42(11)$ & $76(19.9)$ & $118(30.9)$ & $1.36(0.84-2.19)$ & $1.82(0.99-3.34)$ \\
\hline & $9-10$ & 75 (19.6) & $100(26.2)$ & $175(45.8)$ & $1.62(0.92-2.84)$ & $1.35(0.77-2.38)$ \\
\hline & $11-13$ & $42(11)$ & $47(12.3)$ & $89(23.3)$ & 1 & 1 \\
\hline \multirow[t]{2}{*}{ Hand washing habit } & Sometimes & $81(21.2)$ & $131(34.3)$ & $212(55.5)$ & $1.37(0.91-2.07)$ & \\
\hline & Always & $78(20.4)$ & $92(24.1)$ & $170(44.5)$ & 1 & \\
\hline \multirow[t]{2}{*}{ Shoe wearing habit } & Sometimes & $80(20.9)$ & $121(31.7)$ & $201(52.6)$ & $1.17(0.78-1.76)$ & \\
\hline & Always & $79(20.7)$ & $102(26.7)$ & $181(47.4)$ & 1 & \\
\hline \multirow[t]{3}{*}{ Mother education } & Illiterate & $55(14.4)$ & $70(18.3)$ & $125(32.7)$ & $0.69(0.41-1.16)$ & \\
\hline & Primary school & $64(16.8)$ & $79(20.7)$ & $143(37.4)$ & $0.67(0.40-1.11)$ & \\
\hline & Secondary school \& above & $40(10.5)$ & $74(19.4)$ & $114(29.8)$ & 1 & \\
\hline \multirow[t]{2}{*}{ Fingernail cleanness } & Not clean & $47(12.3)$ & $70(18.3)$ & $117(30.6)$ & $0.92(0.59-1.43)$ & \\
\hline & Clean & $112(29.3)$ & $153(40.1)$ & $265(69.4)$ & 1 & \\
\hline
\end{tabular}

Not: ${ }^{*}=p<0.05,{ }^{* *}=p<0.01,{ }^{a}=$ adjusted (multivariate regression model) for family monthly income, meal frequency, parasitic infection, sex and age of students, $@=$ Ethiopian birr 
Table 5 Binary logistic regression analysis of potential risk factors associated with stunting among children at selected primary schools in Bahir Dar, Ethiopia, 2014

\begin{tabular}{|c|c|c|c|c|c|c|}
\hline \multirow[t]{2}{*}{ Risk factors } & \multirow[t]{2}{*}{ Categories } & \multicolumn{2}{|l|}{ Stunting } & \multirow[b]{2}{*}{ Total (\%) } & \multirow{2}{*}{$\begin{array}{l}\text { Crude OR } \\
\text { (Cl 95\%) }\end{array}$} & \multirow{2}{*}{$\begin{array}{l}\text { Adjusted OR } \\
(\mathrm{Cl} 95 \%)^{\mathrm{a}}\end{array}$} \\
\hline & & Yes (\%) & No (\%) & & & \\
\hline \multirow[t]{2}{*}{ Meal frequency } & $\leq 3$ times a day & $34(8.9)$ & $254(66.5)$ & $288(75.4)$ & $4.64(2.68-8.03)^{* *}$ & $5.56(2.97-10.41)^{* *}$ \\
\hline & $>3$ times/day & $36(9.4)$ & $58(15.2)$ & $94(24.6)$ & 1 & 1 \\
\hline \multirow[t]{2}{*}{ Sex of students } & Male & $43(11.3)$ & $150(39.3)$ & $193(50.5)$ & $1.72(1.01-2.92)^{*}$ & $1.76(0.99-3.12)$ \\
\hline & Female & $27(7.1)$ & $162(42.4)$ & $189(49.5)$ & 1 & 1 \\
\hline \multirow[t]{3}{*}{ Age (years) } & $7-8$ & $15(3.9)$ & $103(27)$ & $118(30.9)$ & $1.42(0.73-2.77)$ & $1.28(0.63-2.61)$ \\
\hline & $9-10$ & $30(7.9)$ & $145(38)$ & $175(45.8)$ & $2.68(1.32-5.47)^{*}$ & $3.02(1.41-6.47)^{*}$ \\
\hline & $11-13$ & $25(6.5)$ & $64(16.8)$ & $89(23.3)$ & 1 & 1 \\
\hline \multirow[t]{3}{*}{ Mother education } & Illiterate & $21(5.5)$ & $104(27.2)$ & $125(32.7)$ & $1.72(0.80-3.67)$ & $0.82(0.35-1.94)$ \\
\hline & Primary school & $37(9.7)$ & $106(27.7)$ & $143(37.4)$ & $2.97(1.47-6.01)^{*}$ & $2.25(1.06-4.78)^{*}$ \\
\hline & Secondary school \& above & $12(3.1)$ & $102(26.7)$ & $114(29.8)$ & 1 & 1 \\
\hline \multirow[t]{2}{*}{ Parasitic infection } & Infected & $38(9.9)$ & $162(42.4)$ & $200(52.4)$ & $1.10(0.65-1.85)$ & \\
\hline & Not infected & $32(8.4)$ & $150(39.3)$ & $182(47.6)$ & 1 & \\
\hline \multirow[t]{3}{*}{ Family monthly income (ETB ${ }^{@}$ ) } & $<500$ & $21(5.5)$ & $85(22.3)$ & $106(27.7)$ & $0.53(0.21-1.32)$ & \\
\hline & $500-1500$ & $42(11)$ & $173(45.3)$ & $215(56.3)$ & $0.53(0.23-1.26)$ & \\
\hline & $>1500$ & $7(1.8)$ & $54(14.1)$ & $61(16)$ & 1 & \\
\hline \multirow[t]{2}{*}{ Hand washing habit } & Sometimes & $39(10.2)$ & $173(45.3)$ & $212(55.5)$ & $1.08(0.64-1.83)$ & \\
\hline & Always & $31(8.1)$ & $139(36.4)$ & $170(44.5)$ & 1 & \\
\hline \multirow[t]{2}{*}{ Shoe wearing habit } & Sometimes & $38(9.9)$ & $163(42.7)$ & $201(52.6)$ & $1.08(0.64-1.83)$ & \\
\hline & Always & $32(8.4)$ & 149 (39) & $181(47.4)$ & 1 & \\
\hline \multirow[t]{2}{*}{ Fingernail cleanness } & Not clean & $21(5.5)$ & $96(25.1)$ & $117(30.6)$ & $1.04(0.59-1.82)$ & \\
\hline & Clean & $49(12.8)$ & $216(56.5)$ & $265(69.4)$ & 1 & \\
\hline
\end{tabular}

Not: ${ }^{*}=p<0.05,{ }^{* *}=p<0.01,{ }^{a}=$ adjusted (multivariate regression model) for meal frequency, mother education, sex and age of students, ${ }^{@}=$ Ethiopian birr

were positive for one or more intestinal parasites. This finding is in agreement with other reports from Ethiopia [12], Ruanda[21] and Tanzania [22]. The present study showed a high prevalence of intestinal parasite as compared with 27.2\% [15] and 30\% [23] reported from Ethiopia and Sudan, respectively. In the contrary, a high prevalence of intestinal parasitic infection including 69.1\% [24] and 72\% [25] from Ethiopia, 84.7\% from Burkina Faso, [26] and 90\% from Yemen [27] were reported from school children. These reported differences may be explained by the difference in methods used in the study, level of environmental sanitation, sources of drinking water and educational level of their family.

Potential risk factors associated with intestinal parasitic infection were determined in the present study. Multivariate logistic regression analysis demonstrated that a the presence of intestinal parasitic infections were strongly associated with undernutrition, irregular hand washing habit, unclean finger nails and being born from an illiterate mothers. The contribution of these risk factors to a high prevalence of intestinal parasites was reported from Ethiopia [28, 29] and Saudi Arabia [30]. As noted, undernutrition may lead to reduced immune responses that in turn render the students susceptible to parasitic infection[6].

Polyparasitic infection is a common problem of school children in developing countries. Multiple parasitic infections were recorded in $6.3 \%$ of the students in the present study, which is comparable to other reports from Ethiopia $[12,15]$ and Pakistan [31]. In contrast to this finding high prevalence of multiple parasitosis was reported from school children in the Philippines [6].

In this study, we investigated undernutrition among children based on the three indicators (stunting, underweight and thinness). The overall prevalence of undernutrition among school children was found to be $41.6 \%$, which is in agreement with other reports from Northwest Ethiopia[12]. Unlike the present study, a low prevalence of undernutrition was reported from different parts of Ethiopia [10, 13, 32], Nepal [33] and Burkina Faso [26]. Family monthly income, meal frequency, and parasitic infection were independently predicting undernutrition among school children in this study. A similar association of undernutrition with intestinal parasitic infection[26], meal frequency [13] and family monthly income[10, 34] were reported elsewhere. Parasitic infection particularly helminthes leads to 
Table 6 Binary logistic regression analysis of potential risk factors associated with thinness among children at selected primary schools in Bahir Dar, Ethiopia, 2014

\begin{tabular}{|c|c|c|c|c|c|c|}
\hline \multirow[t]{2}{*}{ Risk factors } & \multirow[t]{2}{*}{ Categories } & \multicolumn{2}{|l|}{ Thinness } & \multirow[b]{2}{*}{ Total (\%) } & \multirow{2}{*}{$\begin{array}{l}\text { Crude OR } \\
\text { (Cl 95\%) }\end{array}$} & \multirow{2}{*}{$\begin{array}{l}\text { Adjusted OR } \\
(\mathrm{Cl} 95 \%)^{\mathrm{a}}\end{array}$} \\
\hline & & Yes (\%) & No (\%) & & & \\
\hline \multirow[t]{3}{*}{ Family monthly income $\left(\mathrm{ETB}^{@}\right)$} & $<500$ & $32(8.4)$ & $74(19.4)$ & $106(27.7)$ & $2.21(1.00-4.88)$ & $2.99(1.28-7.01)^{*}$ \\
\hline & $500-1500$ & $60(15.7)$ & $155(40.6)$ & $215(56.3)$ & $1.97(0.94-4.14)$ & $2.69(1.16-6.26)^{*}$ \\
\hline & $>1500$ & $10(2.6)$ & $51(13.4)$ & $61(16.0)$ & 1 & 1 \\
\hline \multirow[t]{2}{*}{ Parasitic infection } & Infected & $40(10.5)$ & $160(41.9)$ & $200(52.4$ & $2.07(1.30-3.28)^{* *}$ & $1.92(1.15-3.19)^{*}$ \\
\hline & Not infected & $62(16.2)$ & $120(31.4)$ & $182(47.6)$ & 1 & 1 \\
\hline \multirow[t]{2}{*}{ Hand washing habit } & Sometimes & $48(12.6)$ & $164(42.9)$ & $212(55.5)$ & $1.59(1.01-2.51)^{*}$ & $1.02(0.58-1.81)$ \\
\hline & Always & $54(14.1)$ & $116(30.4)$ & $170(44.5)$ & 1 & 1 \\
\hline \multirow[t]{2}{*}{ Shoe wearing habit } & Sometimes & $45(11.8)$ & $156(40.8)$ & $201(52.6)$ & $1.59(1.01-2.52)^{*}$ & $1.52(0.91-1.55)$ \\
\hline & Always & $57(14.9)$ & $124(32.5)$ & $181(47.4)$ & 1 & \\
\hline \multirow[t]{2}{*}{ Meal frequency } & $\leq$ 3times a day & $71(18.6)$ & $217(56.8)$ & $288(75.4)$ & $1.50(0.91-2.49)$ & $1.76(0.95-3.29)$ \\
\hline & $>3$ times/day & $31(8.1)$ & $63(16.5)$ & $94(24.6)$ & 1 & 1 \\
\hline \multirow[t]{3}{*}{ Age (years) } & $7-8$ & $25(6.5)$ & $93(24.3)$ & $118(30.9)$ & $1.49(0.86-2.58)$ & $1.39(0.79-2.47)$ \\
\hline & $9-10$ & $50(13.1)$ & $125(32.7)$ & $175(45.8)$ & $1.62(0.86-3.05)$ & $1.71(0.89-3.29)$ \\
\hline & $11-13$ & $27(7.1)$ & $62(16.2)$ & $89(23.3)$ & 1 & 1 \\
\hline \multirow[t]{3}{*}{ Mother education } & Illiterate & $36(9.4)$ & $89(23.3)$ & $125(32.7)$ & $1.08(0.62-1.91)$ & \\
\hline & Primary school & $35(9.2)$ & $108(28.3)$ & $143(37.4)$ & $0.87(0.49-1.52)$ & \\
\hline & Secondary school \& above & $31(8.1)$ & $83(21.7)$ & $114(29.8)$ & 1 & \\
\hline \multirow[t]{2}{*}{ Fingernail cleanness } & Not clean & $28(7.3)$ & $89(23.3)$ & 117 (30.6) & $1.23(0.75-2.04)$ & \\
\hline & Clean & $74(19.4)$ & $191(50)$ & $265(69.4)$ & 1 & \\
\hline \multirow[t]{2}{*}{ Sex of students } & Male & $54(14.1)$ & $139(36.4)$ & $193(50.5)$ & $1.14(0.73-1.79)$ & \\
\hline & Female & 48 (12.6) & $141(36.9)$ & $189(49.5)$ & 1 & \\
\hline
\end{tabular}

Not: ${ }^{*}=p<0.05,{ }^{* *}=p<0.01,{ }^{a}=$ adjusted (multivariate regression model) for family monthly income, meal frequency, parasitic infection, age, hand washing and shoe wearing habit, ${ }^{@}=$ Ethiopian birr

undernutrition due to competition for essential nutrients as well as endogenous nutrient losses [5]. Hookworm and $A$. lumbricoides infections were the most prevalent helminthes infection in the study. Among the two helminthes, A. lumbricoides infection was strongly associated with undernutrition. However, the contribution of hookworm infection to undernutrition was not observed. This difference may be associated with the intensity of the worm, which was not determined in the present study.

Stunted student accounts for about one-fifth of the school children in the present study. This finding is in agreement with the report from school children in Uganda [35] and different regions of Ethiopia [10, 13, $24]$. In contrast to our finding, high prevalence of stunting were reported in different parts of Ethiopia [12, 36, 37], Burkina Faso [26] and Malaysia [38]. The difference might be associated with source of the study population, food type and frequency, and economic and social factors of the study community. This study showed that stunting was strongly associated with age, meal frequency of students and educational level of the mother, which is in agreement with other studies reported from elsewhere [13, 36, 39]. Stunting is a chronic form of undernutrition, which manifested at the latter age of students. Therefore, it is expected to observe stunting at older age groups compared with younger age groups.

Thinness accounts for about one-fourth of the students participated in the study. A similar proportion of thinness was reported from Ethiopia [24] and the Philippines [6]. This finding was a little bit lower compared to 34-50\% $[12,14,37]$ reported from Ethiopia. In contrast to our finding, a low prevalence of thinness was reported from Burkina Faso [26], Nepal [33], Uganda [35] and eastern Ethiopia [15]. These reported variations of thinness in school children among countries suggest the differences in the level of acute malnutrition and food shortage in the target population. Intestinal parasitic infection and low family monthly income were important predictors of thinness in the present study. A similar association of thinness with low family monthly income [40] and parasitic infections [26] were reported elsewhere. Thinness is an acute form of undernutrition which is directly associated with family monthly income. This is because low-income family may not provide a balanced diet and other nutritional needs of the children. In addition, presence of intestinal parasitic infection such as A. lumbricoides compete for 
Table 7 Binary logistic regression analyses of potential risk factors associated with underweight among children at selected primary schools in Bahir Dar, Ethiopia, 2014

\begin{tabular}{|c|c|c|c|c|c|c|}
\hline \multirow[t]{2}{*}{ Risk factors } & \multirow[t]{2}{*}{ Categories } & \multicolumn{2}{|c|}{ Underweight } & \multirow[b]{2}{*}{ Total (\%) } & \multirow{2}{*}{$\begin{array}{l}\text { Crude OR } \\
\text { (Cl 95\%) }\end{array}$} & \multirow{2}{*}{$\begin{array}{l}\text { Adjusted OR (Cl } \\
95 \%)^{\mathrm{a}}\end{array}$} \\
\hline & & Yes (\%) & No (\%) & & & \\
\hline \multirow[t]{2}{*}{ Parasitic infection } & Infected & $28(9.6)$ & $123(42)$ & $151(51.5)$ & $2.24(1.31-3.84)^{* *}$ & $2.43(1.40-4.22)^{* *}$ \\
\hline & Not infected & $48(16.4)$ & $94(32.1)$ & $142(48.5)$ & 1 & 1 \\
\hline \multirow[t]{2}{*}{ Meal frequency } & $\leq 3$ times a day & $36(12.3)$ & $131(44.7)$ & $167(57)$ & $1.69(1.00-2.86)$ & $1.77(1.03-3.05)^{*}$ \\
\hline & $>3$ times/day & $40(13.7)$ & $86(29.4)$ & $126(43)$ & 1 & 1 \\
\hline \multirow[t]{2}{*}{ Sex of students } & Male & $45(15.4)$ & $102(34.8)$ & $147(50.2)$ & $1.64(0.96-2.78)$ & $1.76(1.02-3.04)^{*}$ \\
\hline & Female & 31 (10.6) & $115(39.2)$ & $146(49.8)$ & 1 & 1 \\
\hline \multirow[t]{3}{*}{ Family monthly income $\left(\mathrm{ETB}^{@}\right)$} & $<500$ & $12(4.1)$ & $41(14)$ & $53(18.1)$ & $0.96(0.42-2.34)$ & \\
\hline & $500-1500$ & $47(16)$ & $120(41)$ & $167(57)$ & $1.29(0.68-2.44)$ & \\
\hline & $>1500$ & $17(5.8)$ & $56(19.1)$ & $73(24.9)$ & 1 & \\
\hline \multirow[t]{3}{*}{ Mother education } & Illiterate & $28(9.6)$ & $66(22.5)$ & $94(32.1)$ & $1.56(0.79-3.06)$ & \\
\hline & Primary school & $29(9.9)$ & $81(27.6)$ & $110(37.5)$ & $1.32(0.68-2.56)$ & \\
\hline & Secondary school \& above & $19(6.5)$ & $70(23.9)$ & 89 (30.4) & 1 & \\
\hline \multirow[t]{2}{*}{ Hand washing habit } & Sometimes & $31(10.6)$ & $100(34.1)$ & $131(44.7)$ & $1.24(0.73-2.11)$ & \\
\hline & Always & $45(15.4)$ & $117(39.9)$ & $162(55.3)$ & 1 & \\
\hline \multirow[t]{2}{*}{ Shoe wearing habit } & Sometimes & $31(10.6)$ & $100(34.1)$ & $131(44.7)$ & $1.24(0.73-2.11)$ & \\
\hline & Always & $45(15.4)$ & $117(39.9)$ & $162(55.3)$ & 1 & \\
\hline \multirow[t]{2}{*}{ Fingernail cleanness } & Not clean & $12(4.1)$ & $41(14)$ & $53(18.1)$ & $1.24(0.61-2.51)$ & \\
\hline & Clean & $64(21.8)$ & $176(60.1)$ & $240(81.9)$ & 1 & \\
\hline \multirow[t]{2}{*}{ Age (years) } & $7-8$ & $25(8.5)$ & $93(31.7)$ & $118(40.3)$ & $1.53(0.88-2.65)$ & \\
\hline & $9-10$ & $51(17.4)$ & $124(42.3)$ & $175(59.7)$ & 1 & \\
\hline
\end{tabular}

Not: ${ }^{*}=p<0.05,{ }^{* *}=p<0.01,{ }^{a}=$ adjusted (multivariate regression model) for parasitic infection, meal frequency and sex of students, ${ }^{@}=$ Ethiopian birr

the existing limited nutrient that further leads to acute undernutrition such as thinness.

The proportion of underweight in the present study was comparable with $28.2 \%$ [39] and 24\% [41] reported from Ethiopia. The prevalence of underweight in the present study was considerably lower than other reports from north-west Ethiopia [12] and Sri Lanka [42]. In contrast to our finding, low prevalence of underweight were reported from Ethiopia [13], Egypt [43] and Uganda [35]. These reported differences of underweight could be associated with variation in the socioeconomic level of the family of the target students. The major determinants of underweight in the present study were intestinal parasitic infection, meal frequency and sex of children. Previous studies have reported the contribution of intestinal parasitic infection [26, 35], being male sex [35] and meal frequency [39] on underweight status.

\section{Conclusion}

The present study revealed that undernutrition and intestinal parasitic infections were serious health problem in school children in our study areas. Family monthly income, meal frequency and intestinal parasitic infection were the major predisposing factors for undernutrition.
Similarly, mother education, hand washing habit, undernutrition and finger cleanness of students were associated with parasitic infection. Therefore, concerted efforts are needed to address the risk factors of intestinal parasitic infection and undernutrition.

\section{Abbreviations}

AOR: Adjusted odd ratio; BAZ: Body mass index-for-age; Cl: Confidence interval; COR: Crude odd ratio; HAZ: Height-for-age; SD: Standard deviation; SPSS: Statistical package for social science; WAZ: Weight-for-age; WHO: World health organization

\section{Acknowledgments}

The author forward special gratitude to Bahir Dar University for the financial support required for this study. In addition, I would like to forward my appreciation to the school community, family/guardians of the students and students participated in the study.

\section{Availability of data and materials}

The datasets used and/or analyzed during this study are available from the corresponding author on reasonable request.

\section{Declarations}

I declared that this research article is my original work and all concerned bodies are well acknowledged. This manuscript is neither used for fulfillments of academic work nor submitted to other journals elsewhere.

\section{Authors' contributions}

TH involved in design and conducts the study, data analysis, interpretation of the findings, and drafts the manuscript. 


\section{Authors' information}

Tamirat Hailegebriel (MSc) working as lecturer position in the department of Biology, Bahir Dar University, P.O. Box 79, Bahir Dar, Ethiopia.

\section{Ethics approval and consent to participate}

The study was approved by the ethical review committee of Bahir Dar University, Ethiopia. The purpose of the study was described to the school community and family/guardians of the student. Written consents were obtained from their parents/guardians with full willingness. The participation of student was on voluntary bases and the student withdraws from the study at any time without any obligation. Students positive for intestinal parasitic infections were treated free of charge and all the information were kept confidentially. The nutritional status of the students was informed to their parents/guardian for better management of their diet.

\section{Consent for publication}

Not applicable

\section{Competing interests}

The authors declare that they have no competing interests.

\section{Publisher's Note}

Springer Nature remains neutral with regard to jurisdictional claims in published maps and institutional affiliations.

\section{Received: 18 October 2017 Accepted: 2 August 2018}

\section{Published online: 13 August 2018}

\section{References}

1. Nutrition Challenges [http://www.who.int/nutrition/challenges/en/. Accessed 20 Aug 2017]

2. de-Onis M, Frongillo EA, Blossner M. Is malnutrition declining? An analysis of changes in levels of child malnutrition since 1980. Bull World Health Organ. 2000:78(10):1222-33.

3. Soil-transmitted helminth infections. [http://www.who.int/mediacentre/ factsheets/fs366/en/. Accessed 27 Aug 2017]

4. Brooker S, Clements AC, Bundy DA. Global epidemiology, ecology and control of soil-transmitted helminth infections. Adv Parasitol. 2006:62:22161 .

5. Nurdiati BS, Sumarni S, Suyoko L, Hakimi M, Winkvist A. Impact of intestinal helminthes infection on anemia and iron status during pregnancy: a community based study in Indonesia, Southeast Asian. J Trop Med Public Health. 2001:32(1):14-22.

6. Papier K, Williams GM, Luceres-Catubig R, Ahmed F, Olveda RM, McManus DP, Chy D, Chau TNP, Gray DJ, Ross AGP. Childhood Malnutrition and Parasitic Helminth Interactions. Clin Infect Dis. 2014;59(2):234-43.

7. Stephenson LS, Latham MC, Ottesen EA. Malnutrition and parasitic helminth infections. Parasitol. 2000;121:S23-38.

8. Mekonnen Z, Meka S, Zeynudin A, Suleman S. Schistosoma mansoni infection and undernutrition among school age children in Fincha'a sugar estate, rural part of West Ethiopia. BMC Research Notes. 2014;7:763.

9. Ezeamama AE, Friedman JF, Acosta LP, Bellinger DC, Langdon GC, Manalo DL, Olveda RM, Kurtis JD, Mcgarvey ST. Helminth infection and cognitive impairment among Filipino children. Am J Trop Med Hyg. 2005;72(5):540-8.

10. Reji P, Belay G, Erko B, Legesse M, Belay M. Intestinal parasitic infections and malnutrition amongst first-cycle primary schoolchildren in Adama, Ethiopia. Afr J Prm Health Care Fam Med. 2011;3(1):198.

11. Bemnet A, Beyene M, Bereket F, Ketema T, Desalegn W, Gizachew Y, Tilahun A, Tomoki Y, Andargachew M, Fusao O, et al. Micronutrient levels and nutritional status of school children living in Northwest Ethiopia. Nutrition J. 2012;11:108.

12. Worku N, Erko B, Torban W, Belay M, Kassu A, Fetene T, Huruy K Malnutrition and intestinal parasitic infections in school children of Gondar, North West Ethiopia. Ethiop Med J. 2009;47(1):9-16.

13. Degarege D, Degarege A, Animut A. Undernutrition and associated risk factors among school age children in Addis Ababa, Ethiopia. BMC Public Health. 2015;15:375.

14. Mahmud MA, Spigt M, Afework MB, Pavon IL, Dinant G, Velasco RB. Risk factors for intestinal parasitosis, anaemia, and malnutrition among school children in Ethiopia. Pathog Glob Health. 2003;107(2):58-65.
15. Tadesse $\mathrm{G}$. The prevalence of intestinal helminthic infections and associated risk factors among school children in Babile town, eastern Ethiopia. Ethiop J Health Dev. 2005;19(2):140-7.

16. Agency CS. Summary and Statistical Report of the 2007 Population and Housing Sensus. Addis Ababa: Federal Democratic Ethiopia Population Sensus Commession; 2008. p. 113.

17. de Onis M, Onyango AW, Borghi E, Siyam A, Nishida C, Siekmann J. Development of a WHO growth reference for school-aged children and adolescents. Bulletin of the World Health Organization. 2007;85:660-7.

18. WHO. WHO AnthroPlus for personal computers software for assessing growth and development the world's children. Geneva: WHO; 2011.

19. WHO Growth Reference data for 5-19 years [http://www.who.int/growthref/ en/. Accessed 27 Aug 2017]

20. Basic laboratory methods in medical parasitology. [http://www.who.int/iris/ handle/10665/40793. Accessed 27 Aug 2017]

21. Emile N, Bosco NJ, Karine B. Prevalence of intestinal parasitic infections and associated risk factors among Kigali Institute of Education students in Kigali, Rwanda. Trop Biomed. 2013;30(4):718-26.

22. Speich B, Marti $H$, Ame S, Ali S, Bogoch I, Utzinger J, Albonico M, Keiser J. Prevalence of intestinal protozoa infection among school-aged children on Pemba Island, Tanzania, and effect of single-dose albendazole, nitazoxanide and albendazole-nitazoxanide. Parasites Vectors. 2013;6:3.

23. Muhajir AEM, Hajissa $K$, Mohamed Z, Aal AA. Prevalence of Intestinal Parasitic Infection among Children in Al-kalakla, Khartoum, Sudan. World Appl Sci J. 2017;35(2):219-22.

24. Abdi M, Nibret E, Munshea A. Prevalence of intestinal helminthicinfections and malnutrition amongschoolchildren of the Zegie Peninsula, northwestern Ethiopia. J Infect Public Health. 2017:10:84-92.

25. Mahmud MA, Spigt M, Bezabih AM, Pavon IL, Dinant G, Velasco RB. Risk factors for intestinal parasitosis, anaemia, and malnutrition among school children in Ethiopia. Pathog Glob Health. 2013;107(2):58-65.

26. Erismann S, Diagbouga S, Odermatt P, Knoblauch AM, Gerold J, Shrestha A, Grissoum T, Kaboré A, Schindler C, Utzinger J, et al. Prevalence of intestinal parasitic infections and associated risk factors among schoolchildren in the Plateau Central and Centre-Ouest regions of Burkina Faso. Parasites Vectors. 2016;9:554.

27. Alwabr GMA, Al-Moayed EE. Prevalence of intestinal parasitic infections among school children of Al-Mahweet Governorate, Yemen. Eur J Biol Res. 2016;6(2):64-73.

28. Ayalew A, Debebe T, Worku A. Prevalence and risk factors of intestinal parasites among Delgi school children, North Gondar, Ethiopia. J Parasitol Vector Biol. 2011;3(5):75-81.

29. Andualem MA. Parasitic Infection and Associated Factors among the Primary School Children in Motta Town, Western Amhara, Ethiopia. Am J Public Health Res. 2014:2(6):248-54

30. Al-Mohammed HI, Amin TT, Aboulmagd E, Hablus HR, Zaza BO. Prevalence of intestinal parasitic infections and its relationship with sociodemographics and hygienic habits among male primary schoolchildren in Al-Ahsa, Saudi Arabia. Asian Pac J Trop Med. 2010;3(11):906-12.

31. Mehraj V, Hatcher J, Akhtar S, Rafique G, Beg MA. Prevalence and Factors Associated with Intestinal Parasitic Infection among Children in an Urban Slum of Karachi. PLOS ONE. 2008;3(11):e3680.

32. Degarege A, Hailemeskel E, Erko B. Age-related factors influencing the occurrence of undernutrition in northeastern Ethiopia. BMC Public Health. 2015;15:108.

33. Joshi SH, Gupta R, Joshi CM, Mahajan V. Determinants of nutritional status of school children-a cross-sectional study in the western region of Nepal. National J Integrated Res Med. 2011;2(1):10-5

34. Tette EMA, Sifah EK, Nartey ET. Factors affecting malnutrition in children and the uptake of interventions to prevent the condition. BMC Pediatrics. 2015; 15:189.

35. Francis L, Kirunda BE, Orach CG. Intestinal Helminth Infections and Nutritional Status of Children Attending Primary Schools in Wakiso District, Central Uganda. Int J Environ Res Public Health. 2012:9:2910-21.

36. Wolde M, Berhan Y, Chala A. Determinants of underweight, stunting and wasting among schoolchildren. BMC Public Health. 2015;15:8.

37. Mekonnen H, Tadesse T, Kisi T. Malnutrition and its Correlates among Rural Primary School Children of Fogera District, Northwest Ethiopia. Nutr Disorders Ther. 2015;S12

38. Rajoo Y, Ambu S, Lim YAL, Rajoo K, Tey SC, Lu CW, Ngui R. Neglected Intestinal Parasites, Malnutrition and Associated Key Factors: A Population 
Based Cross-Sectional Study among Indigenous Communities in Sarawak, Malaysia. PLOS ONE. 2017;12(1):e0170174.

39. Hassen HY, Aregay F, Melkie A, Gezu M, Alayu A, Kefelew T. Chronic Undernutrition and Associated Factors among School Age Children in South West Ethiopia, 2015. J Biol Agric Healthc. 2015;5(21):113-22.

40. Ene-Obong $\mathrm{H}$, Ibeanu V, Onuoha N, Ejekwu A. Prevalence of overweight, obesity, and thinness among urban school-aged children and adolescents in southern Nigeria. Food Nutr Bull. 2012;33(4):242-50.

41. Zerfu M, Mekasha A. Anthropometric assessment of school age children in Addis Ababa. Ethiop Med J. 2006;44(4):347-52.

42. Galgamuwa LS, Iddawela D, Dharmaratne SD, GLS G. Nutritional status and correlated socio-economic factors among preschool and school children in plantation communities, Sri Lanka. BMC Public Health. 2017;17:377.

43. Manyanga T, El-Sayed H, Doku DT, Randall JR. The prevalence of underweight, overweight, obesity and associated risk factors among schoolgoing adolescents in seven African countries. BMC Public Health. 2014;14: 887.

Ready to submit your research? Choose BMC and benefit from:

- fast, convenient online submission

- thorough peer review by experienced researchers in your field

- rapid publication on acceptance

- support for research data, including large and complex data types

- gold Open Access which fosters wider collaboration and increased citations

- maximum visibility for your research: over $100 \mathrm{M}$ website views per year

At $\mathrm{BMC}$, research is always in progress.

Learn more biomedcentral.com/submissions 